02

\title{
Спектроскопия высокого разрешения кристаллов, содержащих ионы переходных металлов (обзор)
}

\author{
(C) М.Н. Попова \\ Институт спектроскопии РАН, \\ 108840 Москва, Троицк, Россия \\ e-mail: popova@isan.troitsk.ru
}

Поступила в редакцию 03.09.2021 г.

В окончательной редакции 03.09.2021 г.

Принята к публикации 10.09.2021 г.

Дан краткий обзор работ по исследованию кристаллов, содержащих ионы металлов с незаполненной $f$ или $d$-оболочками, выполненных в Институте спектроскопии РАН методом фурье-спектроскопии высокого разрешения. Среди основных направлений исследований - сверхтонкая структура в спектрах, изотопические эффекты, роль случайных деформаций, спектроскопия фазовых переходов, низкоразмерные магнетики, связанные электрон-фононные возбуждения.

Ключевые слова: фурье-спектроскопия высокого разрешения, кристаллы с $f$ - и $d$-ионами, сверхтонкая структура, изотопические эффекты, фазовые переходы.

DOI: $10.21883 / \mathrm{OS} .2022 .01 .51882 .43-21$

\section{Введение}

В обзоре приведены основные результаты по исследованию кристаллов, содержащих ионы металлов с незаполненной $f$ - или $d$-оболочками, полученные в Институте спектроскопии РАН (ИСАН) методом фурье-спектроскопии высокого разрешения. Этот метод развивался в ИСАН с самого основания Института в 1968 г., по инициативе первого директора ИСАН Сергея Леонидовича Мандельштама. Во взаимодействии с Центральным конструкторским бюро уникального приборостроения (ЦКБУП) АН СССР были сконструированы и установлены в ИСАН уникальные фурье-спектрометры высокого разрешения УФС-01 и УФС-02. В этой работе принимали участие сотрудники ИСАН и ЦКБУП Г.Н. Жижин, Е.А. Виноградов, А.А. Балашов, В.С. Букреев, Н.Г. Культепин, В.А. Вагин, И.Н. Нестерук, Е.Б. Перминов. Инфракрасный вакуумный фурье-спектрометр УФС-02 работал в области от 50 до $0.8 \mu \mathrm{m} \mathrm{c}$ разрешением до $0.005 \mathrm{~cm}^{-1}$. Такого класса спектрометры в то время были только во Франции, где на них велись исследования атомных и молекулярных спектров. В ИСАН было решено впервые в мире применить фурье-спектроскопию высокого разрешения для исследования конденсированных сред. В 1985 г. были продемонстрированы спектры, которые не удалось бы получить никаким другим методом, а именно спектры кристалла $\mathrm{LiYF}_{4}$ :Но с разрешенной сверхтонкой структурой [1]. С тех пор в ИСАН систематически ведутся исследования различных кристаллов методом фурье-спектроскопии высокого разрешения. В настоящее время в ИСАН функционирует Уникальная научная установка „Мультифункциональная широкодиапазонная спектроскопия высокого разрешения“ (УНУ МШСВР ИСАН) [2]), основу которой составляет фурье-спектрометр высокого разрешения Bruker 125HR. К.Н. Болдырев, С.А. Климин и другие сотрудники Лаборатории фурье-спектроскопии ИСАН существенно расширили возможности коммерческого прибора. На УНУ можно регистрировать спектры пропускания, отражения, люминесценции, диффузного пропускания и отражения, НПВО (в том числе в поляризованном свете, в магнитных полях) в диапазоне $15-30000 \mathrm{~cm}^{-1}$ (от терагерц до ультрафиолета) с разрешением до $0.001 \mathrm{~cm}^{-1}$ во всей спектральной области. Диапазон температур: от $\sim 1.6 \mathrm{~K}$ (гелий с откачкой) до $\sim 450 \mathrm{~K}$ (нагревательная приставка).

Исследования в Лаборатории фурье-спектроскопии ИСАН ведутся в сотрудничестве с теоретиками (в основном это группа Б.3. Малкина в Казанском федеральном университете) и многими российскими и зарубежными научными учреждениями. Можно выделить несколько основных направлений исследований. Это сверхтонкая структура в спектрах, изотопические эффекты, роль случайных деформаций, спектроскопия фазовых переходов, низкоразмерные магнетики, связанные электронфононные возбуждения.

\section{Исследования сверхтонких взаимодействий}

В работах [1,3-14] была зарегистрирована и исследована сверхтонкая структура в спектрах ряда кристаллов, активированных редкоземельными (Р3) ионами. Эти работы, казавшиеся вначале чисто академическими, оказались востребованными в связи с возникновением и интенсивным развитием в последнее десятилетие новой области науки - квантовой информатики. Разрабатываются различные устройства оптической квантовой памяти 
для квантовых повторителей в линиях связи на основе квантовой криптографии, для квантовых компьютеров. Подавляющее большинство уже работающих лабораторных макетов оптической квантовой памяти создано на основе сверхтонких уровней Р3 ионов в кристаллических матрицах. Идет активный поиск новых комбинаций ион-матрица. В связи с этим актуальны исследования сверхтонкой структуры в спектрах перспективных для оптической квантовой памяти материалов.

\section{Изотопические эфффекты}

В работе [15] была обнаружена тонкая структура сверхтонких компонент линий в спектрах поглощения примесных ионов гольмия в кристаллах двойных фторидов лития-иттрия, $\mathrm{LiYF}_{4}$ : Но. Экспериментально было доказано, что эта структура в спектрах одноизотопного элемента гольмия обусловлена изотопическим беспорядком в подрешетке лития и что кристаллическое поле, действующее на ион $\mathrm{Ho}^{3+}$, зависит от изотопического состава по литию в ближайшем окружении иона $\mathrm{Ho}^{3+}$. Из-за разницы масс изотопов ${ }^{6} \mathrm{Li}$ и ${ }^{7} \mathrm{Li}$ амплитуды их колебаний отличаются, и вследствие ангармонизма нулевых колебаний это приводит к смещению положений равновесия ближайших ионов фтора. Теоретическое рассмотрение (Б.З. Малкин) показало, что именно этот механизм статической деформации решетки является доминирующим в образовании изотопической структуры по сравнению с динамическим эффектом, обусловленным электрон-фононным взаимодействием [16]. Изотопическая структура, связанная с изотопическим беспорядком в подрешетке лития в кристалле $\mathrm{LiYF}_{4}$, была также обнаружена и исследована в электронных спектрах других примесных ионов: эрбия [6,17] и тулия $[10,11]$. Развитая теория была использована также для интерпретации спектров отражения кристаллов двойных фторидов лития-иттрия с различным содержанием изотопов лития, ${ }^{6} \mathrm{Li}_{x}{ }^{7} \mathrm{Li}_{1-x} \mathrm{YF}_{4}$ [18]. Работа [19] содержит краткий обзор наших исследований сверхтонкой и изотопической структуры в оптических спектрах кристаллов с Р3 ионами.

Эти работы показали, что изотопический беспорядок вносит существенный вклад в неоднородное расщепление и уширение спектральных линий и стимулировали использование изотопически чистых кристаллов в современных устройствах квантовой информатики.

\section{Неоднородное уширение и тонкая структура спектральных линий, обусловленные случайными деформациями кристаллической решетки}

Вопрос неоднородного уширения спектральных линий является фундаментальным вопросом спектроскопии.
В классической работе Стоунхэма [20] были проанализированы формы неоднородно уширенных линий синглетсинглетных переходов, обусловленные различного типа дефектами. В этой и многих последующих работах использовалась одномерная функция распределения случайных деформаций в изотропном континууме. Такой подход явно не годится для описания неоднородного уширения спектральных линий переходов между уровнями, хотя бы один из которых орбитально вырожден, и в реальных анизотропных кристаллах. В спектрах высокого разрешения Р3 ионов в кристаллических полях кубической [21], тетрагональной $[11,22,23]$ и тригональной [24] симметрии мы обнаружили спектральные линии со специфическим провалом в центре, соответствующие переходам с участием орбитально вырожденных электронных состояний. Была развита микроскопическая теория деформационного уширения и расщепления спектральных линий, включающая вывод обобщенной функции распределения компонент тензора случайных деформаций, индуцированных точечными дефектами в упруго анизотропном континууме (Б.З. Малкин с сотрудниками) [23]. Измерения эволюции формы линий (ширины провалов) с изменением концентрации Р3 ионов позволили получить оценки сил дефектов, сопоставляемых примесным ионам в активированных кристаллах [22,23]. Результаты этой серии работ продемонстрировали возможность использования спектроскопии высокого разрешения для количественной оценки качества оптических материалов и оптимизации режимов их синтеза.

\section{Редкоземельные бораты со структурой хантита}

Большой кластер работ посвящен исследованию соединений из обширного семейства функциональных боратов со структурным типом природного минерала хантита с общей химической формулой $\mathrm{RM}_{3}\left(\mathrm{BO}_{3}\right)_{4}$ $(\mathrm{R}=\mathrm{Y}, \mathrm{La}-\mathrm{Lu} ; \mathrm{M}=\mathrm{Al}, \mathrm{Ga}, \mathrm{Fe}, \mathrm{Cr}, \mathrm{Sc})$. Хантиты кристаллизуются в основном в нецентросимметричной тригональной структуре (оптическая нелинейность, магнитоэлектрические свойства), содержащей цепочки $\mathrm{MO}_{6}$ октаэдров, соединенных изолированными друг от друга $\mathrm{RO}_{6}$-призмами (малое тушение люминесценции $\mathrm{R}^{3+}$ P3 ионов). Это широкозонные диэлектрики. Такие особенности в совокупности с механической прочностью, хорошей теплопроводностью и химической стойкостью делают эти соединения перспективными для использования в качестве лазерных и нелинейных сред вплоть до ультрафиолета. В то время как алюмобораты широко используются в квантовой электронике, галлиевые бораты изучены мало. Мы показали, что их люминесцентные и нелинейные свойства сравнимы с таковыми для алюмоборатов [25].

Хантиты с магнитными ионами $\mathrm{M}^{3+}(\mathrm{Fe}, \mathrm{Cr})$ обладают интересными магнитными свойствами. Мы исследовали 
весь ряд Р3 ферроборатов [26-39]. На основании сведений об энергиях и симметрии штарковских уровней РЗ ионов и их расщеплениях при магнитном упорядочивании, полученных из анализа спектров высокого разрешения, найдены параметры кристаллического поля и обменных взаимодействий [26-32]. Эти параметры использованы для моделирования магнитных свойств Р3 ферроборатов [26-32], киральной структуры электрических квадрупольных моментов [31] и вычисления электрической поляризации [30]. При этом для учета квазиодномерности магнитной подсистемы железа разработана модель (Б.З. Малкин) четырехчастичного кластера из ионов железа - минимальная модель, воспроизводящая свойства цепочки [33]. Выполнены оценки эффективности различных механизмов магнитоэлектрического отклика ферроборатов. Измеренные характеристики спектров высокого разрешения, дополненные расчетами параметров кристаллических полей и штарковской структуры мультиплетов Р3 ионов, позволили определить тип магнитной структуры в магнитоупорядоченной фазе исследованных соединений, причем и в тех случаях, когда невозможно или затруднительно провести исследования магнитного рассеяния нейтронов $[29,30]$. Предсказан эффект киральности индуцированных полем магнитных моментов в парамагнитной фазе ферробората диспрозия [31]. Обнаружен новый эффект - появление при магнитном упорядочении новой линии в спектре поглощения, отвечающей запрещенному переходу в ферроборате празеодима [34]. Обнаружены и исследованы связанные электрон-фононные возбуждения [35-38]. Открыт и объяснен новый эффект, вызываемый резонансным электрон-фононным взаимодействием в антиферромагнитном диэлектрике, помещенном во внешнее магнитное поле, а именно образование щели в спектре электронных возбуждений [37].

Недавняя работа [39] посвящена исследованию низкотемпературных структурных и магнитных фазовых переходов в твердых растворах $\mathrm{Eu}_{1-x} \mathrm{La}_{x} \mathrm{Fe}_{3}\left(\mathrm{BO}_{3}\right)_{4}$. Показано, что температуру структурного перехода и ширину петли гистерезиса можно регулировать, изменяя $x$ (состав), и предложена модель, объясняющая экспериментальные результаты. Продемонстрирована ситуация, когда температура структурного перехода $(12 \mathrm{~K})$ значительно ниже температуры магнитного упорядочения $(32 \mathrm{~K})$.

В отличие от ферроборатов хантиты с другим магнитным ионом, $\mathrm{Cr}^{3+}$, изучены мало. Для них характерны низкотемпературные фазовые переходы и, что сильно осложняет исследования, полиморфизм с присутствием разных структурных фаз в одном кристалле [40].

\section{Низкоразмерные и фрустрированные магнетики}

Изучению низкоразмерных и фрустрированных магнетиков посвящена обширная литература: они инте- ресны прежде всего с точки зрения физики своими необычными чисто квантовыми свойствами. Это спинпайерлсовские переходы в цепочках полуцелочисленных спинов, холдейновская щель в спектре магнитных возбуждений цепочек целочисленных спинов, состояния спинового льда или спиновой жидкости в фрустрированных магнетиках и т.д. Мы выполнили ряд работ по исследованию кристаллов с низкоразмерными или/и фрустрированными магнитными взаимодействиями методом фурье-спектроскопии высокого разрешения.

Исследованию фазовых переходов и кроссовера от одномерного к трехмерному магнетизму в семействе цепочечных никелатов $\mathrm{Y}_{2-x} \mathrm{R}_{x} \mathrm{BaNiO}_{5}(\mathrm{R}-$ редкая земля) - квазиодномерных холдейновских магнетиков (спин ионов $\mathrm{Ni}^{2+} S=1$ ) посвящены работы [41-48]. Тогда как никелат иттрия - идеальный одномерный магнетик (в нем нет магнитного упорядочения, по крайней мере, вплоть до $0.1 \mathrm{~K}$, наблюдается щель Холдейна в спектре магнитных возбуждений, характерная для цепочек целочисленных спинов), замена иттрия на редкую землю приводит к усилению межцепочечного взаимодействия и трехмерному магнитному порядку. Методом P3 зонда были зарегистрированы магнитные фазовые переходы и определены типы магнитных структур в соединениях $\mathrm{R}_{2} \mathrm{BaNiO}_{5}$ [41-44,48]. Сравнение данных измерений с результатами расчетов в рамках развитой Б.3. Малкиным модели обменных зарядов в теории кристаллического поля позволило прийти к заключению, что тип магнитной структуры определяется одноионной магнитной анизотропией Р3 иона $[45,46]$. В инфракрасных спектрах цепочечного никелата гадолиния обнаружен новый тип оптических возбуждений одномерной цепочки спинов $S=1-$ магнитное поглощение с участием фонона [47]. С привлечением результатов расчета динамики решетки рассмотрен возможный механизм формирования спин-фононных возбуждений [47].

В работах [49-57] приведены результаты спектроскопических исследований спин-пайерсовского квазиодномерного магнетика $\mathrm{CuGeO}_{3}$ и соединения $\mathrm{NaV}_{2} \mathrm{O}_{5}$, в котором наблюдается сложный фазовый переход, подобный спин-пайерсовскому, сопровождаемый зарядовым упорядочением. В работах $[58,59]$ на основе спектроскопических данных для $\mathrm{Yb}_{2} \mathrm{Ti}_{2} \mathrm{O}_{7}$ проанализированы параметры кристаллического поля для фрустрированных магнетиков со структурой пирохлора $\mathrm{R}_{2} \mathrm{Ti}_{2} \mathrm{O}_{7}(\mathrm{R}=\mathrm{Tb}$, $\mathrm{Ho}, \mathrm{Er}, \mathrm{Tm}, \mathrm{Yb})$. Физически обоснованные корректные параметры необходимы для интерпретации низкотемпературной магнитной структуры и спиновой динамики пирохлоров. Работы [60-62] посвящены первым результатам исследования новых магнитных соединений $\mathrm{Ln}_{3} \mathrm{CrGe}_{3} \mathrm{Be}_{2} \mathrm{O}_{14}(\mathrm{Ln}=\mathrm{La}, \mathrm{Pr}, \mathrm{Nd})$ с квазиодномерными взаимодействиями в подсистеме хрома и фрустрированными - в Р3 подсистеме. Это первый пример соединений из функционального семейства лангаситов, в которых присутствуют две магнитные подсистемы. 
Следует упомянуть еще одно чрезвычайно интересное магнитное соединение - метаборат меди $\mathrm{CuB}_{2} \mathrm{O}_{4}$, имеющий тетрагональную кристаллическую структуру. Несмотря на простую формулу, структура сложная, с 12 формульными единицами в элементарной ячейке и двумя неэквивалентными позициями для ионов меди $\mathrm{Cu}^{2+}$. При этом в одной из подсистем меди доминируют одномерные магнитные взаимодействия, и она остается неупорядоченной при самых низких температурах. Вторая подсистема меди упорядочивается при температуре около $20 \mathrm{~K}$, ее характеризует чрезвычайно сложная фазовая Н-Т-диаграмма. В нашей работе [63] обнаружен появляющийся при магнитном упорядочении оптический линейный дихроизм в изотропной кристаллографической базовой плоскости кристалла метабората меди. Выяснена природа линейного дихроизма и показано, что он может служить чувствительным, селективным по подрешеткам методом обнаружения фазовых переходов и зондирования магнитных структур. Работа [63] также поставила точку в принципиальной дискуссии о возможности контролировать киральность кристалла с помощью магнитного поля.

\section{Заключение}

Возможности исследований конденсированных сред методом фурье-спектроскопии высокого разрешения далеко не исчерпываются затронутыми областями. Недавно в ИСАН были получены интересные результаты по физике гибридных органо-неорганических перовскитов, различных примесных центров в алмазе и кремнии, магнитоструктурных фазовых переходов в ряде соединений.

\section{Благодарности}

Я признательна своим коллегам - соавторам по публикациям за плодотворную совместную работу.

\section{Конфликт интересов}

Автор заявляет, что у нее нет конфликта интересов.

\section{Список литературы}

[1] N.I. Agladze, M.N. Popova. Solid State Commun., 55, 1097 (1985).

[2] http://www.ckp-rf.ru/usu/508571

[3] Н.И. Агладзе, Е.А. Виноградов, М.Н. Попова. ЖЭТФ, 91 (4), 1210 (1986). [N.I. Agladze, E.A. Vinogradov, M.N. Popova. Sov. Phys. JETP, 64 (4), 716 (1986)].

[4] Н.И. Агладзе, Е.А. Виноградов, М.Н. Попова. Опт. и спектр., 61 (1), 3 (1986). [N.I. Agladze, E.A. Vinogradov, M.N. Popova. Opt. Spectrosc. (USSR), 61 (1), 1 (1986)].

[5] M.N. Popova, N.I. Agladze. Molecular physics, 102, 1315 (2004). DOI: $10.1080 / 00268970410001728618$
[6] M.N. Popova, E.P. Chukalina, B.Z. Malkin, S.K. Saikin. Phys. Rev. B, 61, 7421 (2000). DOI: 10.1103/PhysRevB.61.7421

[7] M.N. Popova, E.P. Chukalina, B.Z. Malkin, E. Antic-Fidancev, P. Porcher, J.P. Chaminade. Phys. Rev. B, 63, 075103 (2001). DOI: 10.1103/PhysRevB.63.075103

[8] J.-P. Wells, R. Glynn, D. Jones, M.F. Reid, M.N. Popova, E.P. Chukalina. Molecular physics, 102, 1367 (2004). DOI: 10.1080/00268970410001728555

[9] D.S. Pytalev, S.A. Klimin, M.N. Popova. Phys. Lett. A, 372, 2332 (2008). DOI: 10.1016/j.physleta.2007.11.053

[10] D.S. Pytalev, S.A. Klimin, M.N. Popova. Phys. Lett. A, 372, 3506 (2008). DOI: 10.1016/j.physleta.2008.01.072

[11] S.A. Klimin, D.S. Pytalev, M.N. Popova, B.Z. Malkin, M.V. Vanyunin, S.L. Korableva. Phys. Rev. B, 81, 045113 (2010). DOI: 10.1103/PhysRevB.81.045113

[12] E.P. Chukalina, D.S. Pytalev, M.N. Popova. Physics Letters A, 375, 3205 (2011). DOI: 10.1016/j.physleta.2011.07.011

[13] D.S. Pytalev, E.P. Chukalina, M.N. Popova, G.S. Shakurov, S.L. Korableva, B.Z. Malkin. Phys. Rev. B, 86, 115124 (2012). DOI: 10.1103/PhysRevB.86.115124

[14] M.N. Popova. Optical Materials, 35, 1842 (2013). DOI: 10.1016/j.optmat.2013.03.013

[15] N.I. Agladze, M.N. Popova, G.N. Zhizhin, V.J. Egorov, M.A. Petrova. Phys. Rev. Lett., 66, 477 (1991). DOI: 10.1103/PhysRevLett.66.477

[16] Н.И. Агладзе, М.А. Корейба, Б.З. Малкин, В.Р. Пекуровский, М.Н. Попова. ЖЭТФ, 104 (6), 4171 (1993). [N.I. Agladze, M.N. Popova, M.A. Koreiba, B.Z. Malkin, V.R. Pekurovskii. JETP, 77 (6), 1021 (1993)]

[17] E.P. Chukalina, M.N. Popova, S.L. Korableva, R.Yu. Abdulsabirov. Phys. Lett. A, 269 (5-6), 348 (2000). DOI: 10.1016/S0375-9601(00)00273-5

[18] E.A. Vinogradov, V.A. Yakovlev, N.N. Novikova, M.N. Popova, S.K. Saikin, B.Z. Malkin. Solid State Commun., 142, 256 (2007). DOI: 10.1016/j.ssc.2007.02.03

[19] М.Н. Попова. Опт. и спектр., $119(4), 541$ (2015). DOI: $10.7868 / \mathrm{S} 0030403415100256$ [M.N. Popova. Opt. Spectr., 119 (4), 544 (2015). DOI: $10.1134 / \mathrm{S} 0030400 \mathrm{X} 15100239]$

[20] A.M. Stoneham. Rev. Mod. Phys., 41, 82 (1969). DOI: $10.1103 /$ RevModPhys.41.82

[21] B.Z. Malkin, D.S. Pytalev, M.N. Popova, E.I. Baibekov, M.L. Falin, K.I. Gerasimov, N.M. Khaidukov. Phys. Rev. B, 86, 134110 (2012). DOI: 10.1103/PhysRevB.86.134110

[22] G.S. Shakurov, E.P. Chukalina, M.N. Popova, B.Z. Malkin, A.M. Tkachuk. Phys. Chem. Chem. Phys., 16, 24727 (2014). DOI: $10.1039 / \mathrm{c} 4 \mathrm{cp} 03437 \mathrm{f}$

[23] B.Z. Malkin, N.M. Abishev, E.I. Baibekov, D.S. Pytalev, K.N. Boldyrev, M.N. Popova, M. Bettinelli. Phys. Rev. B, 96, 014116 (2017). DOI: 10.1103/PhysRevB.96.014116

[24] K.N. Boldyrev, N.M. Abishev, I.E. Mumdzi, S.L. Nikitin, P.J. Deren, B.Z. Malkin, M.N. Popova. Phys. Rev. B, 103, 054103 (2021). DOI: 10.1103/PhysRevB.103.054103

[25] Н.Н. Кузьмин, К.Н. Болдырев, Н.И. Леонюк, С.Ю. Стефанович, М.Н. Попова. Опт. и спектр., 127 (1), 112 (2019). DOI: 10.21883/OS.2019.07.47937.101-19 [N.N. Kuz'min, K.N. Boldyrev, N.I. Leonyuk, S.Yu. Stefanovich, M.N. Popova. Opt. Spectr., 127 (1), 107 (2019). DOI: $10.1134 / \mathrm{S} 0030400 X 19070154]$. 
[26] M.N. Popova, E.P. Chukalina, T.N. Stanislavchuk, E.A. Popova, B.Z. Malkin, A.R. Zakirov, E. Antic-Fidancev, LN. Bezmaternykh, V.L. Temerov. Phys. Rev. B, 75, 224435 (2007). DOI: 10.1103/PhysRevB.75.224435

[27] M.N. Popova, T.N. Stanislavchuk, B.Z. Malkin, L.N. Bezmaternykh. Phys. Rev. B, 80, 195101 (2009). DOI: 10.1103/PhysRevB.80.195101

[28] T.N. Stanislavchuk, M.N. Popova, B.Z. Malkin, L.N. Bezmaternykh. J. Phys.: Condens. Matter, 24, 196002 (2012). DOI: 10.1088/0953-8984/24/19/196002

[29] М.Н. Попова, Е.П. Чукалина, Б.З. Малкин, Д.А. Ерофеев, Л.Н. Безматерных, И.А. Гудим. ЖЭТФ, 145 (1), 128 (2014). DOI: 10.7868/S0044451014010131 [M.N. Popova, E.P. Chukalina, B.Z. Malkin, D.A. Erofeev, L.N. Bezmaternykh, I.A. Gudim. JETP, 118 (1), 111 (2014). DOI: $10.1134 / \mathrm{S} 1063776114010142]$

[30] M.N. Popova, B.Z. Malkin, K.N. Boldyrev, T.N. Stanislavchuk, D.A. Erofeev, V.L. Temerov, I.A. Gudim. Phys. Rev. B, 94, 184418 (2016). DOI: 10.1103/PhysRevB.94.184418

[31] M.N. Popova, E.P. Chukalina, K.N. Boldyrev, T.N. Stanislavchuk, B.Z. Malkin, I.A. Gudim. Phys. Rev. B, 95, 125131 (2017). DOI: 10.1103/PhysRevB.95.125131

[32] M.N. Popova, E.P. Chukalina, D.A. Erofeev, I.A. Gudim, I.V. Golosovsky, A. Gukasov, A.A. Mukhin, B.Z. Malkin. Phys. Rev. B, 103, 094411 (2021). DOI: 10.1103/PhysRevB.103.094411

[33] B.Z. Malkin, E.A. Popova, E.P. Chukalina, A. Jablunovskis, M.N. Popova. Physica Status Solidi-Rapid Research Letters, 14, 1900603 (2020). DOI: 10.1002/pssr.201900603

[34] M.N. Popova, T.N. Stanislavchuk, B.Z. Malkin, L.N. Bezmaternykh. Phys. Rev. Lett., 102, 187403 (2009).

DOI: 10.1103/PhysRevLett.102.187403

[35] K.N. Boldyrev, T.N. Stanislavchuk, A.A. Sirenko, L.N. Bezmaternykh, M.N. Popova. Phys. Rev. B, 90, 121101(R) (2014). DOI: 10.1103/PhysRevB.90.121101

[36] S.A. Klimin, A.B. Kuzmenko, M.A. Kashchenko, M.N. Popova. Phys. Rev. B, 93, 054304 (2016). DOI: 10.1103/PhysRevB.93.054304

[37] K.N. Boldyrev, T.N. Stanislavchuk, A.A. Sirenko, D. Kamenskyi, L.N. Bezmaternykh, M.N. Popova. Phys. Rev. Lett., 118, 167203 (2017). DOI: 10.1103/PhysRevLett.118.167203

[38] М.Н. Попова, К.Н. Болдырев. УФН, $\mathbf{1 8 9}(3), 292$ (2019). DOI: 10.3367/UFNr.2018.06.038413 [M.N. Popova, K.N. Boldyrev. Physics-Uspekhi, 62 (3), 275 (2019). DOI: $10.3367 /$ UFNe.2018.06.038413]

[39] K.N. Boldyrev, V.M. Burlakov, I.A. Gudim, S.Yu. Gavrilkin, M.N. Popova. Phys. Rev. Materials 5, 094414 (2021). DOI: 10.1103/PhysRevMaterials.5.094414

[40] K.N. Boldyrev, N.N. Kuz'min, A.A. Mukhin, V.Yu. Ivanov, E.A. Dobretsova, E.A. Popova, S.Yu. Gavrilkin, N.I. Leonyuk, V.V. Maltsev, B.Z. Malkin, M.N. Popova. Phys. Rev. Materials, accepted (2021).

[41] G.G. Chepurko, Z.A. Kazei, D.A. Kudrjavtsev, R.Z. Levitin, B.V. Mill, M.N. Popova, V.V. Snegirev. Phys. Lett. A, 157 (1), 81 (1991). DOI: 10.1016/0375-9601(91)90413-3

[42] Yu.A. Hadjiiskii, I.V. Paukov, M.N. Popova, B.V. Mill. Phys. Lett. A, 189 (1-2), 109 (1994). DOI: 10.1016/03759601(94)90827-3

[43] M.N. Popova, Yu.A. Hadjiiskii, I.V. Paukov, B.V. Mill. Phys. Lett. A, 203 (5-6), 412 (1995). DOI: 10.1016/03759601(95)00391-F
[44] S.A. Klimin, A.S. Galkin, M.N. Popova. Phys. Lett. A, 376 (23), 1861 (2012). DOI: 10.1016/j.physleta.2012.03.013

[45] M.N. Popova, S.A. Klimin, E.P. Chukalina, B.Z. Malkin, R.Z. Levitin, B.V. Mill, E. Antic-Fidancev. Phys. Rev. B, 68, 155103 (2003). DOI: 10.1103/PhysRevB.68.155103

[46] M.N. Popova, S.A. Klimin, E.P. Chukalina, E.A. Romanov, B.Z. Malkin, E. Antic-Fidancev, B.V. Mill, G. Dhalenne. Phys. Rev. B, 70, 024414 (2005). DOI: $10.1103 /$ PhysRevB.71.024414

[47] S.A. Klimin, A.B. Kuzmenko, M.N. Popova, B.Z. Malkin, I.V. Telegina. Phys. Rev. B, 82, 174425 (2010). DOI: $10.1103 /$ PhysRevB.82.174425

[48] S.A. Klimin, A.S. Galkin, M.N. Popova. J. Alloys Compd., 625, 193 (2015). DOI: 10.1016/j.jallcom.2014.11.129

[49] M.N. Popova, A.B. Sushkov, S.A. Golubchik, A.N. Vasil'ev, L.I. Leonyuk. Phys. Rev. B, 57, 5040 (1998). DOI: 10.1103/PhysRevB.57.5040

[50] М.Н. Попова, А.Б. Сушков, А.Н. Васильев, М. Исобэ, Ю. Уэда. Письма в ЖЭТФ, 65 (9), 711 (1997). [M.N. Popova, A.B. Sushkov, A.N. Vasil'ev, M. Isobe, Yu. Ueda. JETP Lett., 65 (9), 743 (1997). DOI: $10.1134 / 1.567420]$.

[51] S.A. Golubchik, M. Isobe, A.N. Ivlev, B.N. Mavrin, M.N. Popova, A.B. Sushkov, Yu. Ueda, A.N. Vasil'ev. J. Phys. Soc. Japan, 66 (12), 4042 (1997).

[52] A.I. Smirnov, M.N. Popova, A.B. Sushkov, S.A. Golubchik, D.I. Khomskii, M.V. Mostovoy, A.N. Vasil'ev, M. Isobe, Y. Ueda. Phys. Rev. B, 59, 14546 (1999). DOI: 10.1103/PhysRevB.59.14546

[53] M.N. Popova, A.B. Sushkov, S.A. Golubchik, B.N. Mavrin, V.N. Denisov, B.Z. Malkin, A.I. Iskhakova, M. Isobe, Y. Ueda. ЖЭТФ, 115, 2170 (1999). [M.N. Popova, A.B. Sushkov, S.A. Golubchik, B.N. Mavrin, V.N. Denisov, B.Z. Malkin, A.I. Iskhakova, M. Isobe, Y. Ueda. JETP, 88 (6), 1186 (1999). DOI: $10.1134 / 1.558909]$.

[54] М.Н. Попова. УФН, $169(3), \quad 353 \quad$ (1999). DOI: $10.3367 /$ UFNr.0169.199903n.0353 [M.N. Popova, Physics-Uspekhi, 42 (3), 288 (1999). DOI: 10.1070/PU1999v042n03ABEH000532].

[55] M.N. Popova, A.B. Sushkov, S.A. Golubchik, M. Isobe, Y. Ueda. Physica B, 284-288, Part 2, 1617 (2000). DOI: 10.1016/S0921-4526(99)02723-4

[56] M.N. Popova, A.B. Sushkov, S.A. Klimin, E.P. Chukalina, B.Z. Malkin, M. Isobe, Yu. Ueda. Phys. Rev. B, 65, 144303 (2002). DOI: 10.1103/PhysRevB.65.144303

[57] М.Н. Попова, А.Б. Сушков, Е.П. Чукалина, Е.А. Романов, М. Изобэ, Ю. Уэда, ФТТ, 44 (8), 1390 (2002). [M.N. Popova, A.B. Sushkov, E.P. Chukalina, E.A. Romanov, M. Isobe, Y. Ueda. Phys. Solid State 44 (8), 1450 (2002). DOI: 10.1134/1.1501335].

[58] B.Z. Malkin, A.R. Zakirov, M.N. Popova, S.A. Klimin, E.P. Chukalina, E. Antic-Fidancev, Ph. Goldner, P. Aschehoug, G. Dhalenne. Phys. Rev. B, 70, 075112 (2004). DOI: $10.1103 /$ PhysRevB.70.075112

[59] С.А. Климин, М.Н. Попова, Е.П. Чукалина, Б.3. Малкин, А.P. Закиров, Е. Antic-Fidancev, Ph. Goldner, P. Aschehoug, G. Dhalenne. ФTT, 47 (8), 1376 (2005). [S.A. Klimin, M.N. Popova, E.P. Chukalina, B.Z. Malkin, A.R. Zakirov, E. Antic-Fidancev, Ph. Goldner, P. Aschehoug, G. Dhalenne. Phys. Solid State, 47 (8), 1425 (2005). DOI: $10.1134 / 1.2014481]$ 
[60] M.M. Markina, B.V. Mill, G. Pristáš, M. Marcin, S.A. Klimin, K.N. Boldyrev, M.N. Popova. J. Alloys Compd., 779, 380 (2019). DOI: 10.1016/j.jallcom.2018.11.227

[61] S.A. Klimin, B.V. Mill, M.N. Popova. J. Rare Earths, 37, 1250 (2019). DOI: 10.1016/j.jre.2018.12.011

[62] N. Kuzmin, S. Klimin, B. Mavrin, V. Chernyshev, B. Mill, M. Popova. J. Phys. Chem. Solids, 138, 109266 (2020). DOI: $10.1016 /$ j.jpcs.2019.109266

[63] K.N. Boldyrev, R.V. Pisarev, L.N. Bezmaternykh, M.N. Popova, Phys. Rev. Lett., 114, 247210 (2015). DOI: 10.1103/PhysRevLett.114.247210 